\title{
Uso de la tecnología en la formación médica
}

\section{Use of Technology in Medical Training}

\section{Frank Lizaraso Caparólab, Napoleón Paredes Pérez ${ }^{2 c d}$}

En los últimos años, hemos observado en el país, un cambio acelerado en la metodología educativa para el dictado de las diversas asignaturas a lo largo de toda la formación médica; desde el pre al posgrado. Cada vez más, notamos que se consolida el uso de herramientas informáticas y la virtualización del conocimiento, para el logro de las Ilamadas "competencias".

Hoy, se valora e incentiva el autoaprendizaj e dejando poco a poco de lado la conferencia o clase magistral, en la que el profesor es el protagonista, por la enseñanza participativa, convirtiéndose la clase en un diálogo fluido, de intercambio de ideas y valoración de lo significativo. Proceso que desde hace varios años desarrollamos en nuestra Casa de Estudios con equipos y medios informáticos que facilitan el aprendizaje y la evaluación, bajo el lema: Ama lo que haces, aprende como.

Dado el avance acelerado del conocimiento y la tecnología, estamos convencidos que pronto desplazarán a la clase presencial por el uso de la tecnología. Las aplicaciones para tablets, smartphones o "teléfonos inteligentes", cada vez son más accesibles a los alumnos y el aprendizaje se facilita con fotos, videos, podcasts, conferencias en línea y artículos científicos de acceso abierto, herramientas que obligarán a las Escuelas de Medicina a adecuarse a la modernidad del conocimiento, tal como ya viene ocurriendo en algunas Universidades de los Estados Unidos de Norteamérica y Europa (1).

Situación que cada vez se hará más necesaria, especialmente cuando los pacientes también tengan los dispositivos y la costumbre de acceder a la información médica, requiriendo del médico: comentarios y opiniones de manera específica, por lo que observamos el crecimiento y desarrollo de estrategias tecnológicas para facilitar el diagnóstico y el entendimiento de la evolución de las enfermedades (2-4).

Las prácticas y el contacto con el paciente es necesario, pero ya nadie duda del valor de los equipos de simulación y la virtualización de la enseñanza (5), tanto así; que recientemente la Organización Mundial de la Salud valida el aprendizaje virtual con un estudio realizado por el Colegio Imperial de Londres (6).

En esta última década, varias Facultades de Medicina de los Estados Unidos, tienen estudiantes con iPads y smartphones, algunas incluso facilitan fondos para su compra, como la Universidad de Stanford (Programa eStudent) y la Facultad de Medicina de Irvine de la Universidad de California.

Implementar esta nueva forma de enseñar la medicina, tomará su tiempo en nuestro medio, pero ya nosotros lo estamos iniciando. Esta forma de educar, da nuevas posibilidades: teleconferencias en grupo, intercambio de videos y participación a distancia (Second Life), tecnología que la Universidad de San Martín de Porres, ya viene ensayando desde hace algunos años, formando docentes y facilitando una plataforma en USA, para tal efecto.

Será necesario cambiar de manera significativa el currículo y el Plan de Estudios. No se reemplazará el aprendizaje presencial de la anatomía o la práctica clínica, pero con los nuevos aparatos, como el iPad, podrán acceder a contenidos interactivos adicionales y de auto-evaluación, que mejorarán la calidad de su aprendizaje (7). 
En la especialización, también existe evidencia del valor del uso de los medios tecnológicos y la mejor performance del Residente (8).

En conclusión, cada vez será más necesaria la formación con el uso de la tecnología, el reto es dotar de una plataforma segura y contenido adecuado para asegurar la enseñanza de manera continua y sostenida.

\section{REFERENCIAS BIBLIOGRÁFICAS}

1. Ruiz J, Mintzer M, Leipzig R. The Impact of E-Learning in Medical Education. Academic Medicine 2006; 81(3):207-212

2. Park LG, Howie-Esquivel J, Dracup K. A quantitative systematic review of the efficacy of mobile phone interventions to improve medication adherence. J Adv Nurs 2014;70:1932-53.

3. Van Velthoven MH, Li Y, Wang W, Chen L, Du X, Wu Q, et al. (2015) Prevalence of Mobile Phones and Factors Influencing Usage by Caregivers of Young Children in Daily Life and for Health Care in Rural China: A Mixed Methods Study. PLoS ONE 2015; 10(3):1-24.

4. Curioso WH, Mechael PN. Enhancing 'M-health' with south-to-south collaborations. Health Affairs 2010;29: 264-267.

5. Vallance, A., Hemani, A., Fernandez, V., Livingstone, D., McCusker, K., Toro-Troconis, M.
Using virtual worlds for role play simulation in child psychiatry: an evaluation study. Psychiatric Bulletin 2014; 38: 1-7.

6. World Health Organization, Imperial College London. elearning for undergraduate health professional education: a systematic review informing a radical transformation of health workforce development. 1st ed, Edited by Najeeb Al-Shorbaji. Ginebre. 2015.

7. Ranck J. Health information and health care: the role of technology in unlocking data and wellness-a discussion paper. 2011. Disponible desde: http:// www. unfoundation. org/ assets/ pdf/ info-as-care. pdf. Acceso el 9 Marzo 2015.

8. Patel B, Chapman C, Luo N, Woodruff J, Arora V. Impact of Mobile Tablet Computers on Internal Medicine Resident Efficiency. Arch Intern Med. 2012;172(5):436-438.

\footnotetext{
Editor Horizonte Médico

Doctor en Medicina. Médico Cirujano Plástico

Decano de la Facultad de Medicina Humana de la USMP. Lima, Perú.

Director Horizonte Médico

Doctor en Medicina. Médico Ginecólogo Obstetra

Director del Instituto de Investigación, FMH de la USMP. Lima, Perú.
} 